\title{
Superconducting Circuits without Inductors Based on Bistable Josephson Junctions
}

\author{
I. I. Soloviev ${ }^{1,1,2,3,{ }^{*}}$ V. I. Ruzhickiy ${ }^{1,2,3}$ S. V. Bakurskiy, ${ }^{1,2,4}$ N. V. Klenov ${ }^{1,2,3}$ \\ M. Yu. Kupriyanov ${ }^{1}{ }^{1}$ A. A. Golubov, ${ }^{4,5}$ O. V. Skryabina $\odot,{ }^{1,4}$ and V. S. Stolyarov $\circledast^{2,4,6}$ \\ ${ }^{1}$ Lomonosov Moscow State University Skobeltsyn Institute of Nuclear Physics, Moscow 119991, Russia \\ ${ }^{2}$ Dukhov All-Russia Research Institute of Automatics, Moscow 101000, Russia \\ ${ }^{3}$ Physics Department of Moscow State University, Moscow 119991, Russia \\ ${ }^{4}$ Moscow Institute of Physics and Technology, State University, Dolgoprudniy, Moscow Region 141700, Russia \\ ${ }^{5}$ Faculty of Science and Technology and MESA + Institute of Nanotechnology, Enschede 7500 AE, Netherlands \\ ${ }^{6}$ Institute of Solid State Physics RAS, Chernogolovka 142432, Russia
}

(Received 18 February 2021; revised 7 June 2021; accepted 24 June 2021; published 21 July 2021)

\begin{abstract}
Magnetic flux quantization in superconductors allows the implementation of fast and energy-efficient digital superconducting circuits. However, information representation in magnetic flux severely limits the functional density and is a long-standing problem. Here, we introduce the concept of superconducting digital circuits that do not utilize magnetic flux and have no inductors. We argue that neither the use of geometric nor kinetic inductance is promising for the scaling down of superconducting circuits. The key idea of our approach is the utilization of bistable Josephson junctions, allowing the representation of information through the Josephson energy. Since the proposed circuits are composed only of Josephson junctions, they can be called all-Josephson junction (all-JJ) circuits. We present a methodology for the design of circuits consisting of conventional and bistable junctions. We analyze the principles of the circuit's functioning, ranging from simple logic cells to an 8-bit parallel adder. The utilization of bistable junctions in the all-JJ circuits is promising for the simplification of schematics and a decrease of the JJ count, leading to space efficiency.
\end{abstract}

DOI: 10.1103/PhysRevApplied.16.014052

\section{INTRODUCTION}

The promised end to Moore's law [1] nowadays gives rise to "beyond Moore's" technologies. An appropriate alternative to complementary metal oxide semiconductor (CMOS) technology should be fast and scalable, while providing the highest energy efficiency. Superconducting electronics is attractive in this context.

Superconductor technology is known for high clock frequencies, $f_{c} \sim 2-50 \mathrm{GHz}$ [2], and low energy dissipation per logic operation, down to several zJ [3]. The advantage over CMOSs in energy efficiency reaches 2 to 6 orders of magnitude, depending on the logic and algorithm utilized $[2,4-7]$. This is especially valuable for operation at low temperatures. At standard $T=4.2 \mathrm{~K}$, the cost of refrigeration is 1000 times the dissipated energy $[4,8]$. These unique features make superconducting circuits the most promising candidates for developing scalable computing systems that operate across the gradient between room temperature and the temperature of a cryogenic payload. They are suitable for frontier technologies, such as

\footnotetext{
*isol@phys.msu.ru
}

quantum computers, cognitive radio, scalable sensors, and the quantum internet [9].

At the same time, the integration density of superconducting circuits is far lower than that of current CMOSs. Recently demonstrated benchmark circuits for the latest 250- and 150-nm MIT Lincoln Laboratory (LL) processes were shift registers with $7.4 \times 10^{6}$ and $1.3 \times 10^{7}$ Josephson junctions $(\mathrm{JJs}) / \mathrm{cm}^{2}$ circuit density, respectively [10]. With the $4 \mathrm{JJ} / \mathrm{bit}$ cell, the functional density is less than $10 \mathrm{Mbit} / \mathrm{cm}^{2}$, showing the need for improvement. Since there is no direct analog of a CMOS transistor in the superconducting element base [5], scaling to higher density requires the development of special approaches.

The function of superconducting logic circuits is based on the effect of magnetic flux quantization, instead of the modulation of conductance [6,7]. This allows a discrete representation of information in the form of a single-flux quantum (SFQ), $\Phi_{0}=h / 2 e$ (where $h$ is Planck's constant, $e$ is the electron charge). The basic element of broadly used rapid SFQ (RSFQ) [11] logic is a superconducting loop interrupted by Josephson junctions, see Fig. 1(a). If the loop inductance, $L$, is high enough that $I_{c} L \approx \Phi_{0}$ (where $I_{c}$ is the JJ critical current), then a SFQ can be held in the 


\section{Storing element}

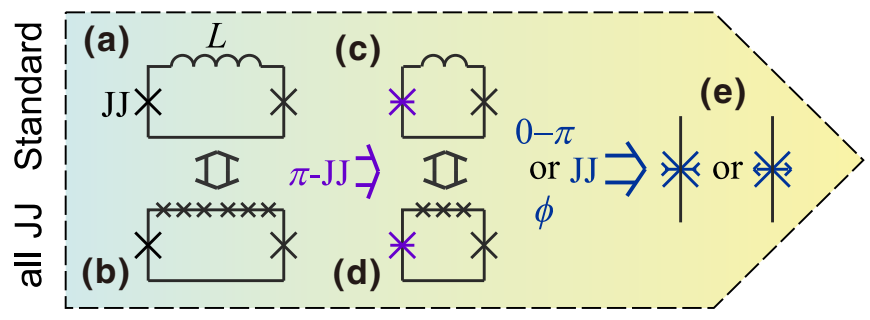

FIG. 1. Storage element based on conventional 0-JJ with (a) and without (b) inductor, $0-\mathrm{JJ}$ and $\pi-\mathrm{JJ}$ with (c) and without (d) inductor, and bistable $0-\pi \mathrm{JJ}$ and $\phi-\mathrm{JJ}$ (e). See Fig. 2 for descriptions of JJ symbols.

loop, representing logic "1," while the absence of a SFQ means logic " 0. ."

Here, we consider different approaches for the miniaturization of the RSFQ basic cell. This includes analysis of the scalability of the inductor, substitution of the inductor by Josephson junctions, and the utilization of magnetic JJs of various kinds. The last of these results in the possibility of replacing the basic cell with a single bistable Josephson junction (see Fig. 1). We complement this by proposing a design methodology for superconducting circuits without inductors based on bistable JJs. Our design is based on a basic block. Modification and complication of its schematic allow us to obtain various logic cells. We demonstrate the validity of the proposed methodology using the design of an 8-bit parallel adder as an example. We conclude the article with a brief discussion of the obtained results and outline further research directions.

\section{MINIATURIZATION OF BASIC CELL}

\section{A. Scaling of inductor}

Theoretical estimation [12] of the maximum density of SFQ-based circuits utilizing geometrical inductance of wires corresponds to already achieved $\sim 10^{7} \mathrm{JJ} / \mathrm{cm}^{2}$. A further decrease of the line width and spacing is problematic because of nearly exponential growth in mutual inductance and cross talk between the inductors [13]. The main approach to shrink the inductor is related to the utilization of kinetic inductance. Here, energy stored in the inductor corresponds to the kinetic energy of the superconducting current, not to the magnetic field around a wire. The critical current of the inductor, $I_{\mathrm{CK}}$, must be greater than that of a Josephson junction, which, in turn, should be much greater than the noise current, $I_{\mathrm{CK}}>I_{c} \gg I_{n}=$ $\left(2 \pi / \Phi_{0}\right) k_{B} T$ (where $k_{B}$ is the Boltzmann constant), to provide a low bit-error rate. The noise current at the operation temperature, $T=4.2 \mathrm{~K}$, is $I_{n} \approx 0.18 \mu \mathrm{A}$. A typical value of the Josephson junction's critical current is $I_{c} \sim 0.1 \mathrm{~mA}$, so that the inductance of a storage cell is $L_{\text {cell }} \approx \Phi_{0} / I_{c} \sim$ $20 \mathrm{pH}$.
For a real type-II superconductor film with width $w$, which is much larger than the coherence length, $w \gg \xi$, the wire critical current, $I_{\mathrm{CK}}$, is a fraction of the GinzburgLandau (GL) depairing critical current, $I_{\mathrm{CK}} \approx \eta I_{\mathrm{GL}}=$ $w d \eta \Phi_{0} /\left(3 \sqrt{3} \xi \mu_{0} \lambda^{2}\right)[14,15]$. Here, $d$ is the thickness of the wire, $\lambda$ is the London magnetic penetration depth, and $\mu_{0}$ is the vacuum permeability. The critical-current reduction [13], $\eta \sim 0.1$, is caused by the entry and motion of Abrikosov vortices from the film edges.

The kinetic inductance of a thin wire is $L_{k}=$ $l \mu_{0} \lambda^{2} /(w d)$, where $l$ is the wire length and $d \ll \lambda$. The required cell inductance is $L_{\text {cell }}=L_{k} \sim l /(w d)$. The critical current of the inductor must also be greater than the $\mathrm{JJ}$, so $I_{c}<I_{\mathrm{CK}} \sim w d$. Therefore, the minimum wire length and its cross-section area are determined by the chosen material and the quality of the wire edges. For a reasonable ratio, $I_{\mathrm{CK}} / I_{c} \geq 4$, and some "dirty" superconductors, such as $\mathrm{MoN}_{x}, \mathrm{Nb}-\mathrm{Ti}-\mathrm{N}$, or $\mathrm{NbN}$, with high kinetic inductance (where $\lambda \approx 500 \mathrm{~nm}, \xi \approx 10 \mathrm{~nm}, \xi / \eta=44 \mathrm{~nm}$ [13]), the wire's geometric parameters are $l=\left(I_{\mathrm{CK}} / I_{c}\right) 3 \sqrt{3} \xi / \eta \geq$ $0.9 \mu \mathrm{m}$ and $w d=l \mu_{0} \lambda^{2} / L_{\text {cell }} \geq 14000 \mathrm{~nm}^{2}$.

If the wire's width is equal to the minimum feature size of standard SFQ5ee MIT LL process technology, $w=350$ $\mathrm{nm}$, the wire's thickness is $d \geq 40 \mathrm{~nm}$, so that the wire's cross-section aspect ratio is $w / d \leq 8.75$. The area of the kinetic inductor is $w l \geq 0.32 \mu \mathrm{m}^{2}$, which is close to the typical area of a Josephson junction, $\pi w^{2} \approx 0.4 \mu \mathrm{m}^{2}$. Implementation of the inductor requires about 2.5 squares while the value of inductance per square is $L_{k} \square \leq 8 \mathrm{pH}$. The obtained numbers show that the utilization of kinetic inductance is a good solution for current technological processes.

However, the convenience of using the kinetic inductance at smaller scales is questionable. For example, a reduction of the inductor width to $w=90 \mathrm{~nm}$ requires an increase in its thickness to $d \approx 160 \mathrm{~nm}$ (if the length is $l=0.9 \mu \mathrm{m})$ to preserve its critical current. Here, the wire's cross-section aspect ratio is $w / d \approx 0.56$, which presents difficulties for fabrication. At the same time, the inductance per square becomes 4 times smaller, so that implementation of the inductor is much less effective at the new scale. A safe threshold for kinetic inductor scaling is about $w=\left(I_{\mathrm{CK}} 3 \sqrt{3} \xi \mu_{0} \lambda^{2} / \eta \Phi_{0}\right)^{1 / 2} \approx 120 \mathrm{~nm}$, which provides a square cross section of the wire. Based on these estimations, we conclude that basic cell scaling requires further research into alternative methods.

\section{B. Superconducting loop without inductor}

If a SFQ is inside the standard cell shown in Fig. 1(a), the Josephson phase of one of the junctions is close to $2 \pi n$ (where $n$ is an integer), while the phase of another one is about $2 \pi(n-1)$, so that the total phase increment in the circuit is $2 \pi$. These Josephson phase values correspond to minima of the 
(a)

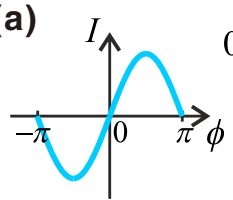

0-JJ

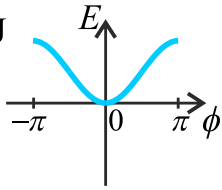

(b)

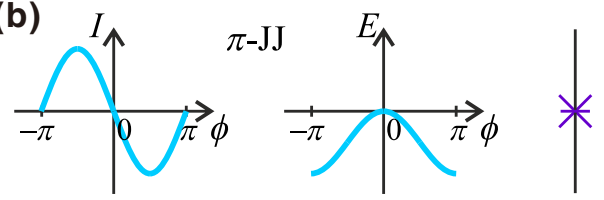

(c)

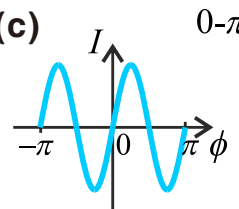

$0-\pi(2 \phi)$

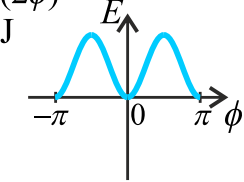

(d)

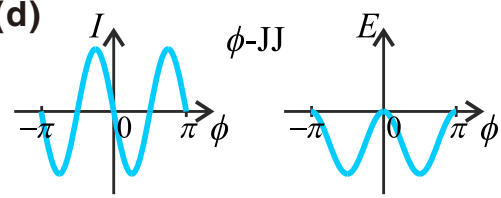

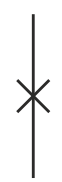

Cell model
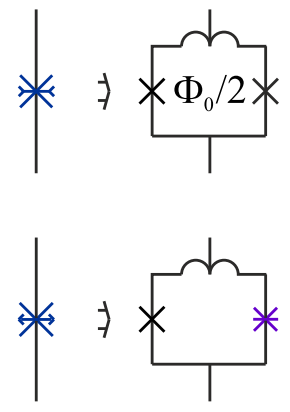

FIG. 2. Current-phase relation, $I(\phi)$; energy-phase relation, $E(\phi)$; and symbols of $0-\mathrm{JJ}(\mathrm{a}), \pi-\mathrm{JJ}(\mathrm{b}), 0-\pi \mathrm{JJ}(\mathrm{c})$, and $\phi$-JJ (d). CPR and EPR of $0-\pi$ and $\phi$-JJ are presented for the case of the suppressed first harmonic of their CPRs. Cell models are presented for $0-\pi(2 \phi)$ and $\phi-J J$.

conventional superconductor-insulator-superconductor JJ (0-JJ hereafter) energy-phase relation (EPR), $E / E_{J 0}=$ $1-\cos (\phi)$, where $\phi$ is the Josephson phase, and $E_{J 0}=$ $I_{c} \Phi_{0} / 2 \pi$ is the Josephson energy, see Fig. 2(a). This EPR corresponds to a sinusoidal current-phase relation (CPR), $I=I_{c} \sin (\phi)$, where $I$ is the current flowing through the junction.

Josephson junctions in the storage cell can be connected by a stack of JJs instead of an inductor [16,17], see Fig. 1(b). Such a cell can be called an all-Josephsonjunction (all-JJ) circuit. If the critical currents of the JJs in a stack are identical, the phase drop for each of them is $2 \pi / N$, where $N$ is the number of JJs in the stack. To prevent the JJs from switching, this phase drop should be less than $\pi / 2$ (in accordance with the 0-JJ CPR). Reliable operation of circuits is supposed to be provided with a phase drop of about $\pi / 3$ [16]. This gives six JJs in a stack that can be implemented, e.g., using two 3-JJ stacks [17].

\section{C. $\pi$ Josephson junction}

The issue of a rather large number of JJs in a stack can be mitigated by the introduction of a JJ with a ferromagnetic interlayer in its weak-link region, providing the $\pi$-phase shift of its CPR, $I=I_{c} \sin (\phi+\pi)(\pi-J J$ hereafter $)[18,19]$. The CPR and EPR of $\pi-J J$ are presented in Fig. 2(b). The $\pi-\mathrm{JJ}$ symbol is a cross with a dash in the middle. With a $\pi-\mathrm{JJ}$ in a loop, the phase increment corresponding to the

(a)

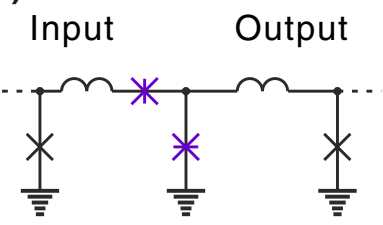

(b)

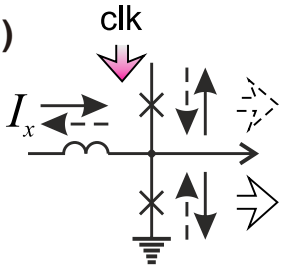

FIG. 3. (a) Two-loop circuit with $\pi$-JJs. (b) Balanced comparator [11].

magnetic half-flux quantum already exists in the cell. The cell inductance can be reduced correspondingly, and the number of JJs in the substituting stack can be decreased down to three, see Figs. 1(c) and 1(d) [20-28]. In the RSFQ toggle ( $\mathrm{T}$ ) flip-flop, it is possible even to completely substitute the inductor with a $\pi-J J$ [20-22]. Unfortunately, this cannot be a general solution for all circuits. The constraints of this method come from the persistent current arising due to the $\pi$-JJ's inherent phase shift. This current flows out into the neighboring cells and may alter their power supplies and disrupt their operation. The direction of this current is determined by the circuit state, and thus, cannot be compensated for by constant-bias-current adjustment.

A design methodology utilizing two $\pi$-JJs connected in series is proposed to circumvent this issue [24]. Figure 3(a) illustrates the idea. Here, the Josephson phase of each of the $\pi$-JJs is about $\pi+2 \pi n$, while that of the $0-\mathrm{JJ}$ in the input circuit is about $2 \pi n$; combined, the phase shift is $2 \pi n$, allowing a state with $n=0$, and thus, zero phase shift and zero current [see also Figs. 2(a) and 2(b)]. This allows the safe connection of the circuit input to conventional RSFQ cells. At the same time, the $\pi+2 \pi n$ phase drop on the central $\pi-\mathrm{JJ}$ means that the $0-\mathrm{JJ}$ of the output loop is outside the minimum potential energy. Minimization of the total energy of the $\pi$ - and 0-JJs leads to states with a noticeable current in the output loop, which can have opposite directions. This provides the opportunity to read out the circuit state by using the conventional balanced comparator shown in Fig. 3(b) [11]. The latter is composed of two JJs connected in series, one of which is switched by the clock (clk) pulse, depending on the direction of the measured current, $I_{x}$. Many widely used cells, such as a delay (D) flip-flop and nondestructive read-out (NDRO) [24], or AND and OR logic cells [25], can be designed within the framework of this approach.

\section{Bistable Josephson junctions}

The main requirement for a storage cell is the existence of two stable states, with and without an SFQ inside. However, the desired bistability can even be obtained with a single bistable Josephson junction. In this case, the storage 
element of the circuits reduces to this a single $\mathrm{JJ}$, and its state does not relate to a SFQ.

The bistability corresponds to the existence of the second harmonic in the JJ's CPR, $I=A \sin (\phi)+B \sin (2 \phi)$. If $|B|>A / 2$, the EPR has a double-well shape [29]. In the case of the positive second-harmonic amplitude, $B>0$, the JJ's energy minima are located at phase values of zero and $\pi$ [29]. This junction is, therefore, called the $0-\pi \mathrm{JJ}$ [30-32]. Notably, the disappearance of the first harmonic here leads to a doubling of the CPR. In this case, the JJ is called $2 \phi$-JJ [33-37]. If the second-harmonic amplitude is negative, $B<0$, the JJ's energy minima are symmetrically located around zero at $\pm \phi$ phases, where $|\phi| \leq \pi / 2$ [29]. This JJ is called $\phi$-JJ [32,38-42]. The CPR and EPR of $0-\pi$ and $\phi$-JJs for the case of the suppressed first harmonic are presented in Figs. 2(c) and 2(d). The symbols of the JJs are similar to that of the $\pi-\mathrm{JJ}$ but with arrows added to the ends of the central dash, which are directed inside or outside for $0-\pi$ and $\phi$-JJ, respectively.

The considered bistable junctions are relatively recently developed types of JJs. They have not yet been introduced into digital superconducting technology. While the optimal method for their fabrication is a technological challenge, they can already be modeled using cells composed of standard junctions [43]. The model of the $2 \phi$-JJ is a superconducting loop with two 0-JJs and a half-flux quantum (HFQ) applied to the cell, see Fig. 2(c) [29]. The cell has a small but finite inductance. A smaller inductance results in a smaller cell critical current, and thus, the cell's CPR is closer to a double-sine function. Here, the decrease of the first-harmonic amplitude is caused by the circulating current induced by the HFQ, while the second-harmonic amplitude, and hence, the effective critical current of the junction, is determined by the inductance.

In a very similar way, a $\phi$-JJ can be modeled by a cell with 0 - and $\pi$-JJs, see Fig. 2(d) [29]. It is shown that a Josephson transmission line (JTL) composed of such cells (modeling $\phi$-JJs) is capable of transmitting a HFQ [2628]. The move toward manipulation with a HFQ instead of a SFQ makes the HFQ circuits more power efficient than any other superconducting logic circuits [28]. Notably, the small inductance in the cell model can be substituted by 0-JJs to obtain the all-JJ circuit [29].

The utilization of bistable JJs seems promising with respect to power and space efficiency. Below, we propose a design methodology for circuits without inductors based on bistable $2 \phi$-JJs. The latter are chosen because their cell model contains only conventional 0-JJs, see Fig. 2(c). Since the proposed design requires only two types of JJs, conventional 0 -JJs and bistable $2 \phi$-JJs, this simplifies the experimental verification of prototype circuits. The information bit in the circuits is represented as the presence or absence of a $2 \pi$ superconducting phase change. This phase change can be transferred along the circuit by the application of a bias current.

\section{DESIGN METHODOLOGY}

\section{A. Basic block}

The dynamics of the $2 \phi-\mathrm{JJ}$, as well as the $0-\mathrm{JJ}$, is described by the well-known resistively shunted junction model with capacitance (RSJC) [44] (see also the Supplemental Material [29] for details):

$$
i=A \sin \phi+B \sin 2 \phi+\alpha \dot{\phi}+\ddot{\phi},
$$

where current flowing through the junction, $i$, and the amplitudes of the CPR harmonics, $A$ and $B$, are normalized to the critical current of a reference junction, $I_{c}$. Dots indicate derivatives with respect to time, $t$, normalized to the inverse plasma frequency, $\tau=t \omega_{p}$, which is determined by the constants of a certain fabrication process, $\omega_{p}=\sqrt{2 \pi j_{c} / \Phi_{0} c}$, where $j_{c}$ is the critical current density of Josephson junctions and $c$ is their specific capacitance. $\alpha=\omega_{p} / \omega_{c}$ is the Josephson junction's damping coefficient, and $\omega_{c}=2 \pi I_{c} R / \Phi_{0}$ is the junction's characteristic frequency; $R$ is the junction's resistance in the normal state.

Each type of junction considered has only one harmonic dominating in the CPR. Therefore, the dominating harmonic determines the critical current of the junction. Since the circuits consist only of JJs and each JJ has only two parameters, the critical current and the damping coefficient [29], these parameters determine the circuit dynamics. The former reflects the strength of superconducting coupling, which determines the potential barrier for JJ switching, while the latter reflects the duration of switching.

The proposed circuits are based on the basic block designed in accordance with the methodology presented in Ref. [24]: the input loop is in a current-less state, while the different phases of the $2 \phi-\mathrm{JJ}$ correspond to different currents circulating in the output loop.

The input loop is composed of one $0-\mathrm{JJ}$ and two $2 \phi$-JJs, see Fig. 4(a). The $2 \pi n$ phase drop on the $0-\mathrm{JJ}$ is distributed between the $2 \phi$-JJs, meaning the phase drop is multiples of $\pi$ on each of them. This corresponds to the current-less state of the loop due to the CPRs of the JJs used, see Fig. 2. Therefore, the loop can be connected to other circuits from the side of the 0 -JJ.

A circulating current in the circuit with two 0-JJs and one $2 \phi$-JJ, see Fig. 4(b), corresponds to a $\pi+2 \pi n$ phase drop on the latter. At the same time, the $2 \pi n$ phase drop on the $2 \phi$-JJ provides the current-less state. A combination of the two considered loops [Figs. 4(a) and 4(b)] forms our basic block, see Fig. 4(d).

In our simulations, we utilize all-Josephson junction (all-JJ) transmission lines (TLs) to apply and read out superconducting $2 \pi$ phase changes to or from the circuits. These are the JTLs in which each inductor is substituted for a single Josephson junction, see the all-JJTL cell in Fig. 4(c). Parameters of the circuits, as well as a 
(a)

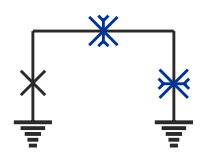

(d)

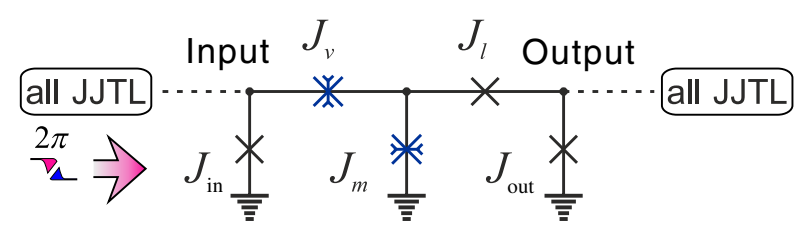

FIG. 4. Interface circuits to change (a) and read-out (b) $2 \phi$-JJ state. (c) all-JJTL basic cell. (d) Schematic of basic block with interface all-JJTLs.

detailed description of their dynamics, are presented in the Supplemental Material [29].

The logic state of the basic block corresponds to the Josephson phase of the main $2 \phi$-JJ, $J_{m}$, located in the center of the circuit [Fig. 4(d)]. $J_{m}$ parameters are depicted on the $(B, \alpha)$ plane in Fig. 5(a) by a star as a reference point. The superconducting phase-change wave enters the basic block through junction $J_{\text {in }}$ and can exit through junction $J_{\text {out }}$. The interconnecting junctions, $J_{v}$ and $J_{l}$, serve as the input and output valves in the wave-transfer process, respectively.

The block possesses four modes of operation, depending on the parameters of the input valve junction, $J_{v}(B, \alpha)$, as presented in Fig. 5(a). In a trivial case where junction $J_{v}$ is sufficiently "weaker" and "faster" than the main junction, $J_{m}$, the input $2 \pi$ phase-change wave switches $J_{\text {in }}$ and exits through $J_{v}$ (mode 1). In the opposite case, the input wave passes through $J_{m}$, switching it twice, and exits through $J_{\text {out }}$ (mode 2).

More interesting modes of operation are obtained with a mixed ratio of parameters of the $2 \phi$-JJs. If the input valve junction is weaker than the main junction, it starts to switch first, but if its switching takes a relatively long time, the main junction also has time to switch [see Fig. 5(b)]. In this way, the incoming $2 \pi$ phase change is transformed into the $\pi$ phase drops on the two $2 \phi$-JJs. Since the output junction, $J_{\text {out }}$, has a $2 \pi$ periodic CPR, every second $2 \pi$ phase-change wave passes through the basic block. Here, the block operates as a digital-frequency divider, like a $\mathrm{T}$ flip-flop (mode 3), see Figs. 5(b) and 5(c).

Simultaneous switching of the $2 \phi$-JJs occurs over a relatively wide range of ratios of parameters (including the inverse ratio with the corresponding swap in the sequence of switching). Interestingly, an increase of the input valve junction's critical current results in another operation regime. Here, leakage of the interface all-JJTL's bias current into $J_{\text {out }}$ provides its periodic switching, if
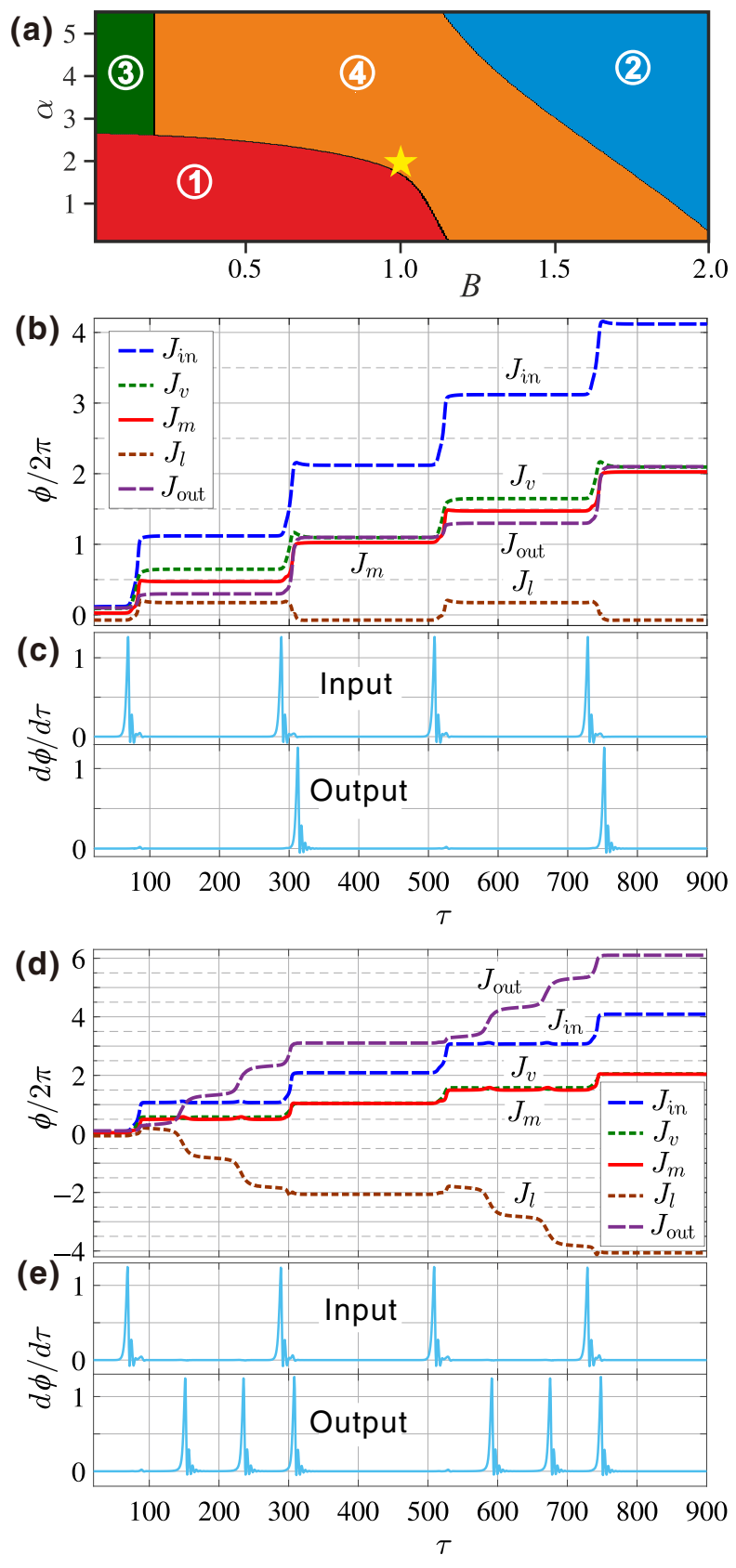

FIG. 5. (a) Input valve junction, $J_{v}$ [shown in Fig. 4(d)], parameters $(B, \alpha)$ corresponding to different modes of basic block operation: 1 , terminator; 2 , transmission line; 3 , digital frequency divider; 4, oscillator. Main junction, $J_{m}$, parameters are depicted by a star ( $\star$ ). (b) Dynamics of phases of Josephson junctions and (c) voltage pulses $(d \phi / d \tau)$ corresponding to phase-change waves in the input and output all-JJTLs in mode 3 for $B_{v}=0.1, \alpha_{v}=5$. (d),(e) Same as before, corresponding to mode $4\left(B_{v}=0.5\right.$, $\left.\alpha_{v}=5\right)$.

the $J_{m}$ phase is about $\pi+2 \pi n$. This switching of $J_{\text {out }}$ can be turned on and off by the switching of logic states of the basic block. In this mode 4, the block operates as a conventional SFQ-to-dc converter [11], see Figs. 5(d) and 5(e). 


\section{B. Controlled readout of $2 \phi-\mathbf{J J}$ states}

Controlled readout of the circuit's logic state can be performed if the output of the basic block is designed as a balanced comparator [see Fig. 3(b) [11]]. In this case, another 0-JJ, $J_{r}$, is connected to the output loop, accordingly, see Fig. 6(a). A read $2 \pi$ phase-change wave is applied to the pair of junctions $J_{r}$ and $J_{\text {out }}$.

The NDRO is realized when the processes in the output loop do not significantly affect $J_{m}$. At the same time, the $J_{m}$ phase is increased by about $\pi$ with every $2 \pi$ phasechange wave coming from the input loop. The circuit's operation is close to that of modes 3 and 4 considered above, but here the input wave never passes to the output. The direct passages of waves from the input to the output and back are blocked by making the output valve junction, $J_{l}$, "weak" and "fast" compared with the neighboring junctions, $J_{m}$ and $J_{\text {out }}$. The dynamics of Josephson junctions and voltage pulses corresponding to the propagation of the phase-change waves in the input, read, and output all-JJTLs are presented in Figs. 6(b) and 6(c).

One can switch from NDRO operation to the destructive read-out (DRO) by changing the parameters of the output valve junction, $J_{l}$, namely, making it slower. The DRO is implemented when only the first input $2 \pi$ phasechange wave increases the $J_{m}$ phase by about $\pi$. Another $\pi$ increase is only available with the read wave. Since $J_{m}$ is connected to $J_{l}$, the slower phase increase at the point of their connection allows $J_{v}$ to switch twice with every input $2 \pi$ phase-change wave, if the $J_{m}$ phase is about $\pi+2 \pi n$, see the Supplemental Material [29]. At the same time, with a slowdown of $J_{l}, J_{m}$ has time to switch from the $\pi+2 \pi n$ state, while the $J_{\text {out }}$ phase increases by $2 \pi$. The DRO cell operates as a D flip-flop, see Figs. 6(d) and 6(e).

Notably, read data can be easily inverted. This is achieved by a simple swap of the connection order of $J_{r}$ and $J_{\text {out }}$, see Fig. 6(f). In this case, $J_{m}$ and $J_{\text {out }}$ are connected to the ground through $J_{r}$. Thus, the stationary distribution of the currents and dynamics of the junctions change, so that the circuit parameters require an additional adjustment, see the Supplemental Material [29]. Simulations of the dynamics of NDRO and DRO cells with output inversion are presented in Figs. 6(g) and 6(h) and Figs. 6(i) and 6(j), correspondingly. The DRO cell here performs synchronous data inversion, which is the NOT logic operation.

\section{Example of a cell design}

We present only a simple combination of input and output circuits to write and read the logic state encoded in a $2 \phi$-JJ phase so far. The functionality of a cell can be increased by connecting additional branches. For example, the NDRO output joined with the DRO cell forms a NDRO cell with separate inputs to set or reset the latch, see Fig. 7(a). An additional output interface circuit providing the NDRO is circled by a dotted line. Josephson junctions of this circuit are marked by capital " $R$ " in their subscripts. The designation of other Josephson junctions are the same as those given in Fig. 6(a). The $2 \pi$ phasechange wave entering the cell through $J_{\text {in }}$ switches the logic state by increasing the $J_{m}$ phase by $\pi$, while that entering the cell through $J_{r}$ resets the state. The critical current of the Josephson junction connecting the NDRO branch to the DRO cell is small to decouple these parts.

In the design of this cell, we divide all JJs into "fast" and "slow" ones, with a correspondingly small or large value of the damping coefficient, see Fig. 7(c). After an optimization procedure, we find that the working margins of the bias currents, as well as of the parameters of the Josephson junctions, are greater than $\pm 20 \%$ during low-speed tests, see Figs. 7(b)-7(d). The margins are expected to shrink as the operation frequency increases.

We add a small parasitic inductance in series to every Josephson junction of the considered circuit to examine its effect. The normalized inductance value is $l=$ $2 \pi I_{c} L / \Phi_{0}=0.5\left(1.65 \mathrm{pH}\right.$ for $\left.I_{c}=0.1 \mathrm{~mA}\right)$. While the values of parameters become clearly shifted, after an additional optimization procedure, the working margins are restored to above $\pm 20 \%$. This illustrates the importance of the careful extraction of parameters of the circuits from the layout as well as the overall robustness of the proposed design approach to the existence of parasitic inductors.

\section{Benchmark logic circuit design}

We verify the validity of the proposed design methodology through the design of an 8-bit parallel adder as an example. The basic element of this adder is a half adder (HA) presented in Fig. 8(a). Here, the phase of the main $2 \phi$-JJ, $J_{m}$, is changed by every $2 \pi$ phase-change wave coming from data lines $a$ or $b$ through $J_{v(a, b)}$ junctions. The interface circuit of the output sum $\left(J_{l s}, J_{r s}, J_{s}\right)$ provides destructive readout of the $J_{m}$ state. The interface circuit of the output carry $\left[J_{l c}, J_{c(a, b)}, J_{c}\right]$ provides a nondestructive readout of the $J_{m}$ state before it is changed by the data phase-change waves. Therefore, the carry readout is asynchronous with respect to the clock. Notably, data can flow sequentially or simultaneously to the HA cell. In the last case, the output junction of the carry branch, $J_{c}$, is switched due to the total effect of the two $2 \pi$ phase-change waves. This is similar to the operation of the RSFQ AND cell [11]. Parameters of the half-adder JJs are presented in Table II within the Supplemental Material [29].

Notably, just a single $2 \phi$-JJ is sufficient here to provide data storage. This is in contrast to the RSFQ HA, where insensitivity to the data delay comes at the cost of doubling the SFQ storage circuit [45]. Thus, the number of JJs in the presented schematic is even smaller than that in the RSFQ counterpart, despite the substitution of inductors for JJs. 
(a)

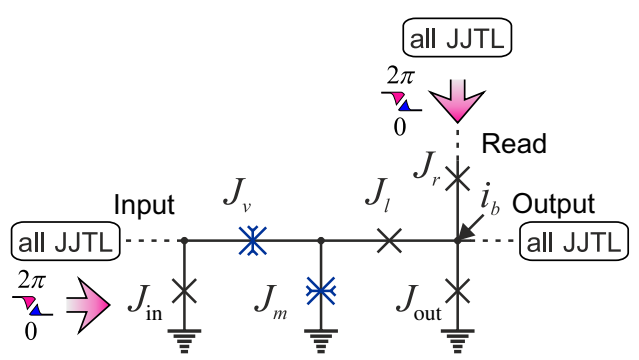

(b)

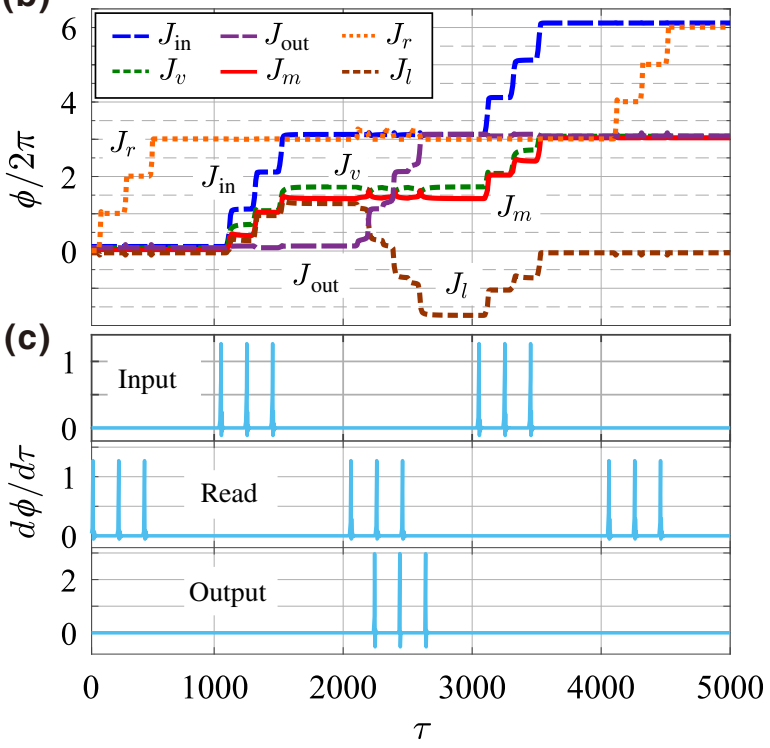

(d)
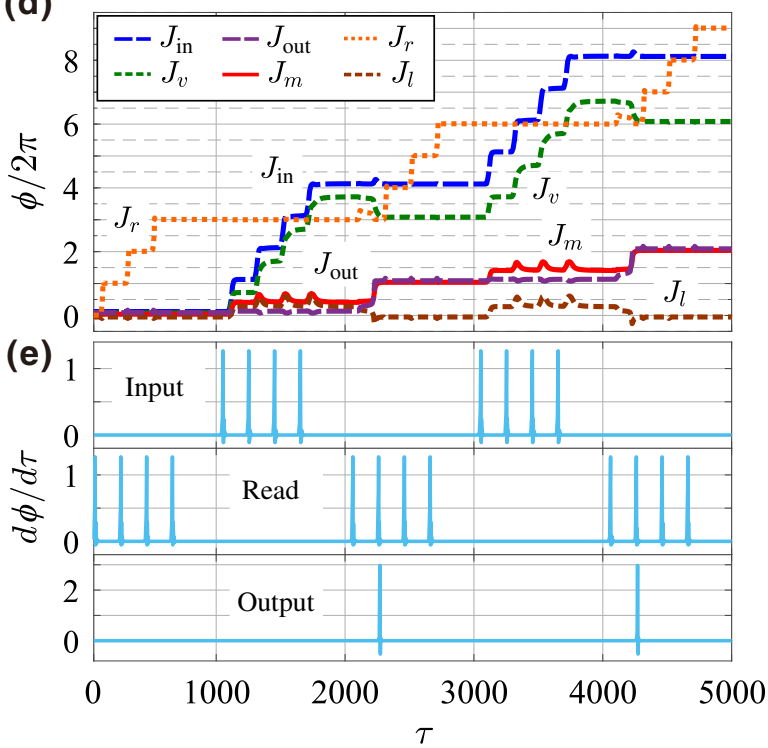

(f)

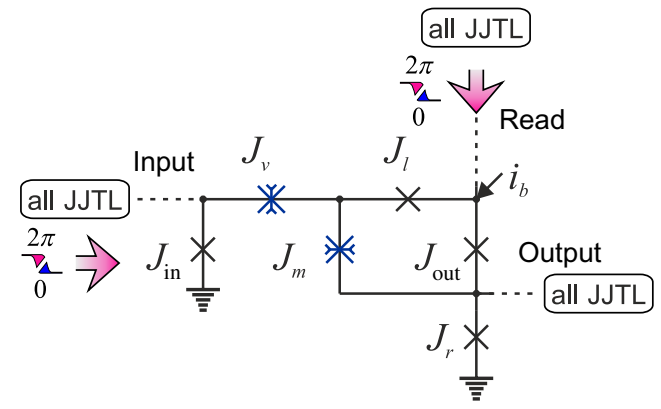

(g)
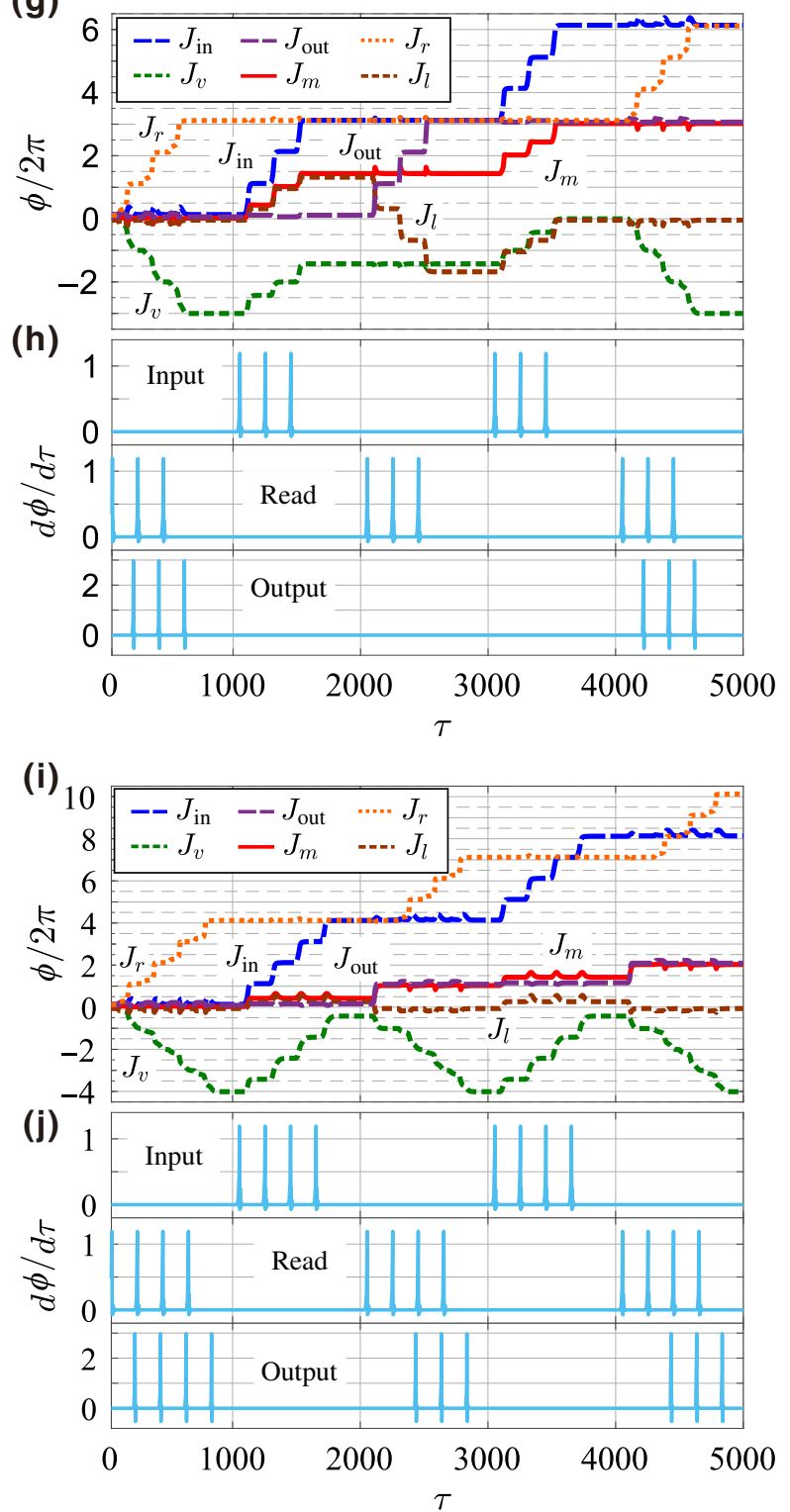

FIG. 6. (a) all-JJ cells based on basic block with controlled readout (NDRO and DRO) of main $2 \phi$-JJ states without (a) and with (f) inversion of read data. Dynamics of phases of Josephson junctions and voltage pulses, corresponding to propagation of phase-change waves $(d \phi / d \tau)$ in the input, read, and output all-JJTLs for NDRO (b),(c); DRO (d),(e); NDRO with output inversion (g),(h); and DRO with output inversion (i),(j) cells. Parameters of Josephson junctions are presented in Table I within the Supplemental Material [29].

An 8-bit ripple-carry-wave pipeline adder schematic is presented in Fig. 8(b). Its energy-efficient RSFQ (ERSFQ [46]) counterpart was proposed earlier as a process benchmark [47]. For an $N$-bit circuit, one needs $2 N-1$ HA cells connected, in our case, by all-JJTLs and all-JJ confluence buffers (CBs). The total number of JJs in the 
(a)

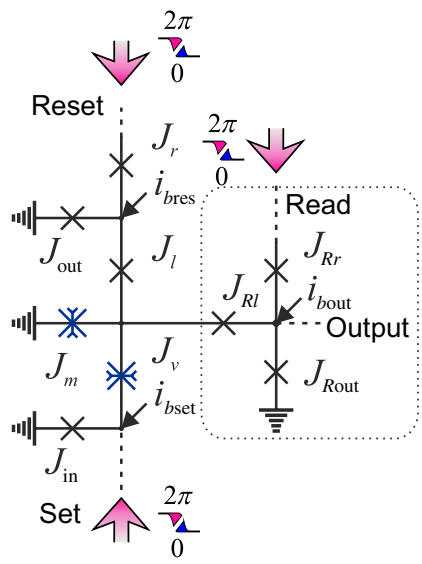

(b)

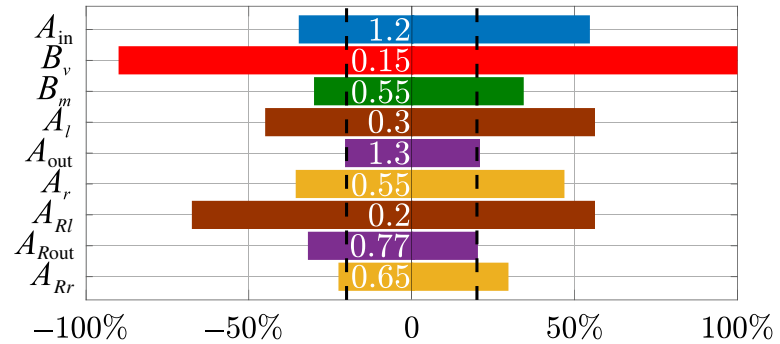

(c)

Critical current margins

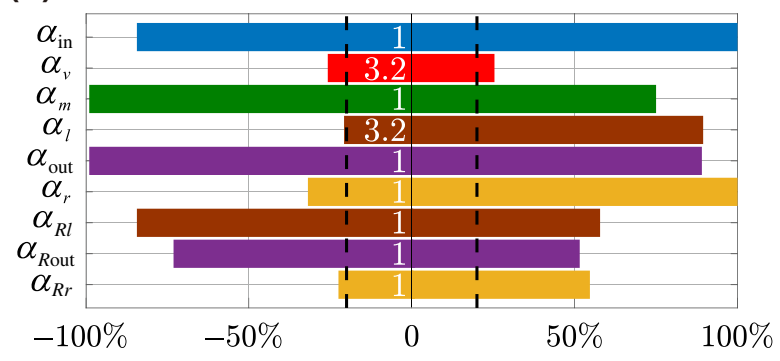

(d)

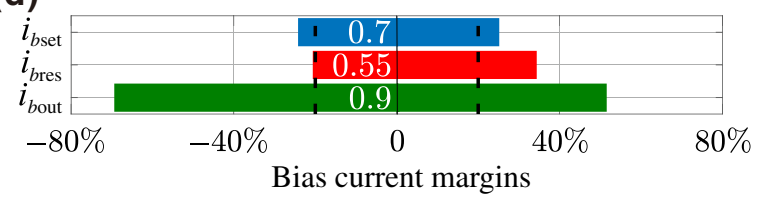

FIG. 7. (a) Schematic of NDRO cell with separate inputs to set or reset the latch formed from a combination of DRO cell with additional NDRO branch (latter is circled by a dotted line). Working margins of critical currents (b), damping coefficients (c), and bias currents (d). Optimal values of parameters are shown in corresponding panels. $\pm 20 \%$ boundaries are shown by vertical dotted lines.

adder is 362 with $150 \mathrm{JJs}$ in HAs (10 JJs per HA) and $212 \mathrm{JJs}$ in all-JJTLs and CBs. An additional $150 \mathrm{JJs}$ are in the clock distribution network. The summation operation is performed in a single clock cycle [47], with a total time delay (normalized to the inverse plasma frequency) of $\tau_{\Sigma}=275$. The output data front is aligned, meaning that least significant and most significant bits are produced simultaneously.
The circuit's operation is simulated with various inputs. Voltage pulses corresponding to the phase-change waves in the $a$ and $b$ data branches, as well as in the sum branches, are shown in Figs. 8(c)-8(e), respectively. The last panel in Fig. 8(e) shows the output carry. The first example presents the summation of two randomly chosen numbers, $a=00100111$ (39) and $b=01010011$ (83), which results in the sum $=01111010$ (122). The second example corresponds to the longest propagation of carry, $a=00000001$ (1) and $b=11111111$ (255), which results in the sum $=$ 00000000 and generation of the output carry (256).

\section{DISCUSSION}

With the presented utilization of $2 \phi$-JJs, one can easily mimic the behavior of RSFQ circuits due to a certain $2 \phi$ JJ CPR. However, other types of bistable junctions are also suitable. Using $0-\pi \mathrm{JJs}$ instead of $2 \phi$-JJs in the basic block would result in some shrinkage of the operation margins caused by a shallower depth of the potential well at $\phi=$ $\pi$. Using $\phi$-JJs with potential-energy minima at $|\phi| \leq \pi / 2$ would additionally lead to some finite current in the output interface circuit in both states of the $\phi$-JJ. Nevertheless, correct circuit operation is possible using these alternative implementations.

A nonzero first harmonic in the CPR provides two critical currents of the bistable JJ. This is due to different heights of potential barriers between the EPR minima. The difference in critical currents gives an opportunity to read out the state directly from the junction [41]. This could serve for further simplification of the schematics.

Logic states 0 and 1 correspond to zero and $2 \pi$ phase changes in the presented circuits. At the same time, the operation of the cells is based on the transformation of the $2 \pi$ phase change into $\pi$ phase drops on the bistable JJs. One can consider an option for more complex information processing with the propagation of $\pi$ phase-change waves. This includes the possibility of ternary logic-circuit design. Three options for the logical states can be associated with zero, $\pi$, and $2 \pi$ phase changes. Manipulation with $\pi$ phase-change waves instead of $2 \pi$ ones can additionally increase the power efficiency [28].

The utilization of bistable JJs eliminates the need for SFQ storage, and hence, the necessity for technological implementation of the quantizing inductance. The inductor can be used as an interconnecting element or not used at all. The latter leads to the fact that the circuit's characteristics are determined only by the parameters of the Josephson junctions. Our results show that the all-JJ circuits can be designed using just a few values of JJ damping, which is promising for their implementation. While circuits without inductors can be implemented with more conventional 0and $\pi$-JJs, the utilization of bistable Josephson junctions significantly decreases the total $\mathrm{JJ}$ count. 
(a)

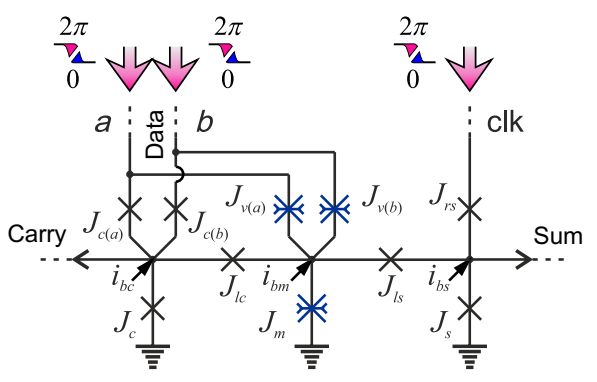

(b)

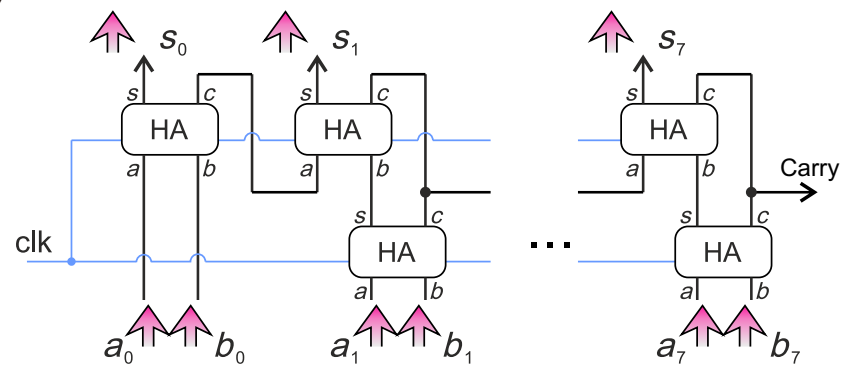

(c)

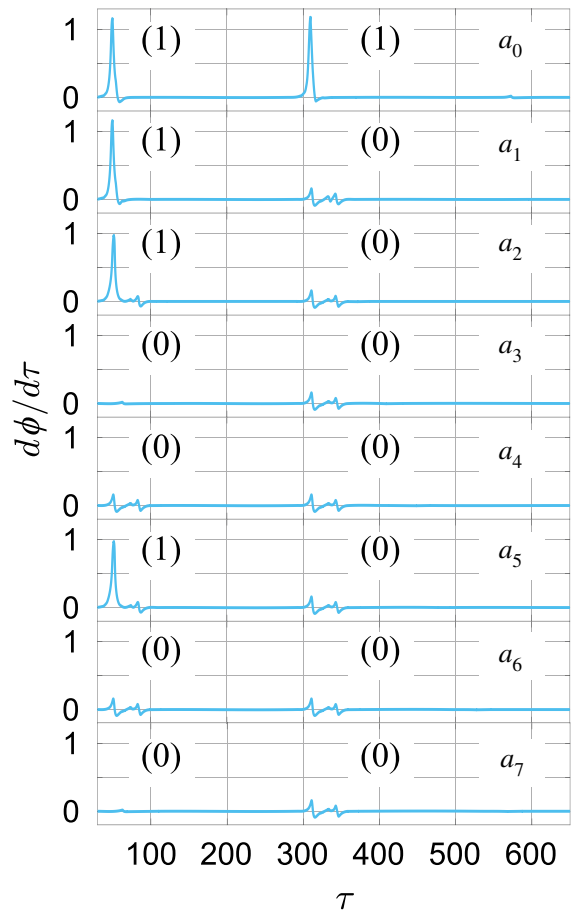

(d)

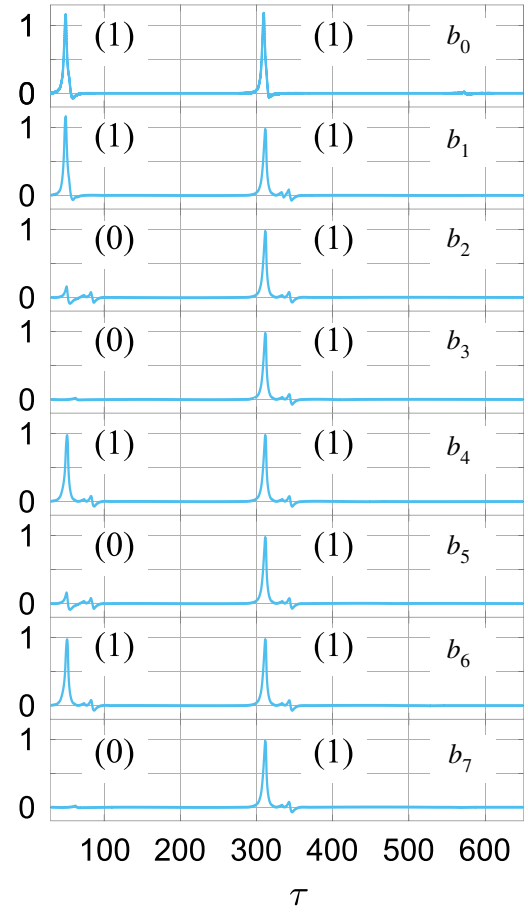

(e)

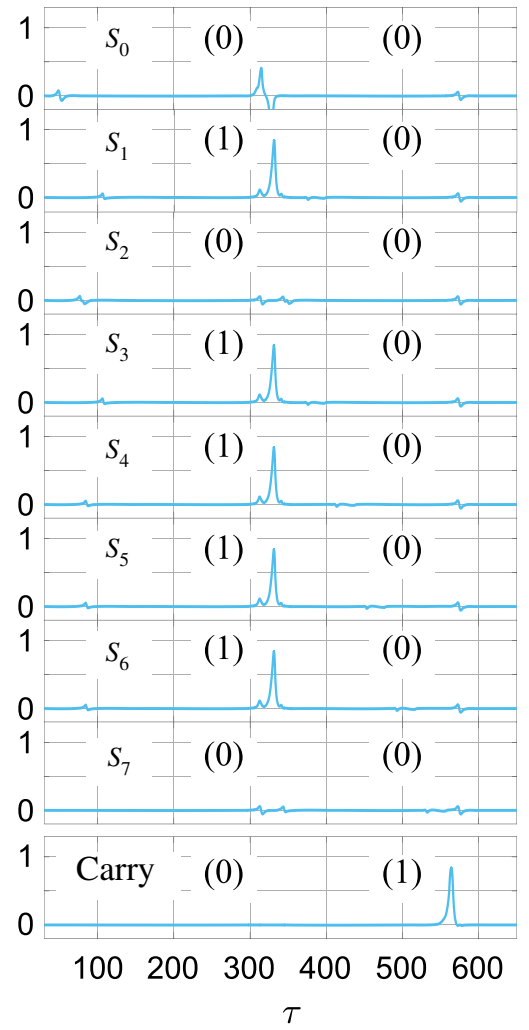

FIG. 8. (a) all-JJ half-adder schematic. (b) 8-bit ripple-carry adder schematic. Voltage pulses corresponding to phase-change waves in data branches $a$ (c) and $b$ (d), and sum branches (e); the bottom panel in the column presents the output carry.

We show that the all-JJ cells can be connected by allJJTLs. However, the utilization of passive transmission lines (PTLs) looks more promising for long-range connections. It is shown that PTLs are suitable for high-density routing with low cross talk, allowing fast data transfer and precision-timing design of the circuits $[48,49]$. The cells can even be connected through PTLs only, although, with a relatively large width of PTLs, this option poses certain limitations on the integration density.

\section{CONCLUSION}

We consider different options for the implementation of the basic storage element of superconducting circuits. We argue that the use of inductors in conventional designs presents technological problems for scaling of the circuit. The utilization of a bistable Josephson junction as a storage element appears to be promising in this context. In this case, magnetic flux is not used as the physical representation of information. This eliminates the requirements imposed for inductors, including the possibility of completely removing them. We present a design methodology for the circuits without inductors based on bistable Josephson junctions. The methodology is used in the design of various basic cells, such as a controlled oscillator analogous to a conventional SFQ-to-dc converter, T flip-flop, D flip-flop, NDRO, NOT logic, and a half adder. A more complex benchmark logic circuit of an 8-bit parallel adder is designed as well. The working margins of the all-JJ NDRO cell with small parasitic inductances in 
low-speed tests exceed $\pm 20 \%$ in simulations. The cell is designed using only a couple of values of the JJ's damping coefficient, which is favorable for its implementation. The design of the half-adder cell shows that the total number of JJs in the all-JJ circuits can be less than that in the standard design. We conclude that the proposed utilization of bistable Josephson junctions is promising with respect to eliminating the need for quantizing inductance; possible simplification of the all-JJ circuit schematics; and a reduction of total JJ count, leading to space efficiency. The search for technological routes to bistable JJ fabrication and the development of information-processing methods using circuits based on such junctions are urgent tasks in this area of research.

\section{ACKNOWLEDGMENTS}

The authors are grateful to D. S. Holmes for fruitful discussions. The development of the all-JJ circuit design methodology is performed under support from Grant No. 20-12-00130 of the Russian Science Foundation. A review of the scalability of the superconducting inductor is supported by a grant from the President of the Russian Federation Grant No. MD-186.2020.8. The design of an 8-bit parallel adder is supported by the RFBR, Grant No. 19-32-90208. V.I.R. acknowledges support from a Basis Foundation scholarship. N.V.K. and M.Yu.K. are grateful to the Interdisciplinary Scientific and Educational School of Moscow University "Photonic and Quantum Technologies. Digital Medicine.”

[1] G. E. Moore, in IEEE International Solid-State Circuits Conference, 2003. Digest of Technical Papers. ISSCC (IEEE, San Francisco, CA, USA, 2003).

[2] N. Takeuchi, Y. Yamanashi, and N. Yoshikawa, Energy efficiency of adiabatic superconductor logic, Supercond. Sci. Technol. 28, 015003 (2015).

[3] N. Takeuchi, Y. Yamanashi, and N. Yoshikawa, Measurement of $10 \mathrm{zJ}$ energy dissipation of adiabatic quantum-fluxparametron logic using a superconducting resonator, Appl. Phys. Lett. 102, 052602 (2013).

[4] D. S. Holmes, A. L. Ripple, and M. A. Manheimer, Energyefficient superconducting computing - power budgets and requirements, IEEE Trans. Appl. Supercond. 23, 1701610 (2013).

[5] I. I. Soloviev, N. V. Klenov, S. V. Bakurskiy, M. Y. Kupriyanov, A. L. Gudkov, and A. S. Sidorenko, Beyond Moore's technologies: Operation principles of a superconductor alternative, Beilstein J. Nanotechnol. 8, 2689 (2017).

[6] O. A. Mukhanov, Energy-efficient single flux quantum technology, IEEE Trans. Appl. Supercond. 21, 760 (2011).

[7] Q. P. Herr, A. Y. Herr, O. T. Oberg, and A. G. Ioannidis, Ultra-low-power superconductor logic, J. Appl. Phys. 109, 103903 (2011).
[8] E. P. DeBenedictis, New design principles for cold, scalable electronics, http://www.debenedictis.org/erik/Cryo_FPGA_ 2LAL/DPfC_51.pdf.

[9] M. Bhushan, et al., IRDS 2020: Cryogenic electronics and quantum information processing, part of IEEE international roadmap for devices and systems (2020), https://irds.ieee.org/editions/2020.

[10] S. K. Tolpygo, V. Bolkhovsky, R. Rastogi, S. Zarr, E. Golden, T. J. Weir, L. M. Johnson, V. K. Semenov, and M. A. Gouker, in Applied Superconductivity Conference 2020 (2020), Vol. 14, https://snf.ieeecsc.org/issues/snfissue-no49-march-2021.

[11] K. K. Likharev and V. K. Semenov, RSFQ logic/memory family: A new Josephson-junction technology for subterahertz-clock-frequency digital systems, IEEE Trans. Appl. Supercond. 1, 3 (1991).

[12] S. K. Tolpygo, V. Bolkhovsky, R. Rastogi, S. Zarr, A. L. Day, T. J. Weir, A. Wynn, and L. M. Johnson, in 2017 16th International Superconductive Electronics Conference (ISEC) (2017), p. 1.

[13] S. K. Tolpygo, V. Bolkhovsky, D. E. Oates, R. Rastogi, S. Zarr, A. L. Day, T. J. Weir, A. Wynn, and L. M. Johnson, Superconductor electronics fabrication process with $\mathrm{MoN}_{x}$ kinetic inductors and self-shunted Josephson junctions, IEEE Trans. Appl. Supercond. 28, 1100212 (2018).

[14] V. L. Ginzburg and L. D. Landau, On the theory of superconductivity, Zh. Experim. i Teor. Fiz. 20, 1064 (1950) (Transl.: in V. L. Ginzburg: On Superconductivity and Superfluidity, A Scientific Autobiography (Springer, Berlin, Germany, 2009), p. 113).

[15] M. Y. Kupriyanov and V. F. Lukichev, Temperature dependence of pair-breaking current in superconductors, Fiz. Niz. Temp. 6, 445 (1980).

[16] S. K. Tolpygo, Superconductor digital electronics: Scalability and energy efficiency issues, Low Temp. Phys. 42, 361 (2016).

[17] M. A. Castellanos-Beltran, D. I. Olaya, A. J. Sirois, P. D. Dresselhaus, S. P. Benz, and P. F. Hopkins, Stacked Josephson junctions as inductors for single flux quantum circuits, IEEE Trans. Appl. Supercond. 29, 1300705 (2019).

[18] V. V. Ryazanov, V. A. Oboznov, A. Y. Rusanov, A. V. Veretennikov, A. A. Golubov, and J. Aarts, Coupling of Two Superconductors through a Ferromagnet: Evidence for a $\pi$ Junction, Phys. Rev. Lett. 86, 2427 (2001).

[19] A. A. Golubov, M. Y. Kupriyanov, and E. Il'ichev, The current-phase relation in Josephson junctions, Rev. Mod. Phys. 76, 411 (2004).

[20] A. V. Ustinov and V. K. Kaplunenko, Rapid single-flux quantum logic using $\pi$-shifters, J. Appl. Phys. 94, 5405 (2003).

[21] T. Ortlepp, A. Ariando, O. Mielke, C. J. M. Verwijs, K. F. K. Foo, H. Rogalla, F. H. Uhlmann, and H. Hilgenkamp, Flip-flopping fractional flux quanta, Science 312, 1495 (2006).

[22] M. I. Khabipov, D. V. Balashov, F. Maibaum, A. B. Zorin, V. A. Oboznov, V. V. Bolginov, A. N. Rossolenko, and V. V. Ryazanov, A single flux quantum circuit with a ferromagnet-based Josephson $\pi$-junction, Supercond. Sci. Technol. 23, 045032 (2010).

[23] T. Ortlepp, A. Ariando, O. Mielke, C. J. M. Verwijs, K. F. K. Foo, A. Andreski, H. Rogalla, F. H. Uhlmann, and H. 
Hilgenkamp, RSFQ circuitry using intrinsic $\pi$-phase shifts, IEEE Trans. Appl. Supercond. 17, 659 (2007).

[24] Y. Yamanashi, S. Nakaishi, A. Sugiyama, N. Takeuchi, and N. Yoshikawa, Design methodology of single-fluxquantum flip-flops composed of both 0 - and $\pi$-shifted Josephson junctions, Supercond. Sci. Technol. 31, 105003 (2018).

[25] Y. Yamanashi, S. Nakaishi, and N. Yoshikawa, Simulation of the margins in single flux quantum circuits containing $\pi$ shifted Josephson junctions, IEEE Trans. Appl. Supercond. 29, 1301805 (2019).

[26] T. Kamiya, M. Tanaka, K. Sano, and A. Fujimaki, Energy/space-efficient rapid single-flux-quantum circuits by using $\pi$-shifted Josephson junctions, IEICE Trans. Electron. E101-C, 385 (2018).

[27] D. Hasegawa, Y. Takeshita, K. Sano, M. Tanaka, A. Fujimaki, and T. Yamashita, in 2019 IEEE International Superconductive Electronics Conference (ISEC) (2019), p. 1.

[28] F. Li, Y. Takeshita, D. Hasegawa, M. Tanaka, T. Yamashita, and A. Fujimaki, Low-power high-speed half-flux-quantum circuits driven by low bias voltages, Supercond. Sci. Technol. 34, 025013 (2021).

[29] See the Supplemental Material at https://link.aps.org/supple mental/10.1103/PhysRevApplied.16.014052 for details on the CPRs of bistable Josephson junctions, their cell models, and our numerical calculations of circuits.

[30] E. Goldobin, D. Koelle, and R. Kleiner, Ground states and bias-current-induced rearrangement of semifluxons in 0- $\pi$ long Josephson junctions, Phys. Rev. B 67, 224515 (2003).

[31] A. Zenchuk and E. Goldobin, Analysis of ground states of $0-\pi$ long Josephson junctions, Phys. Rev. B 69, 024515 (2004).

[32] S. V. Bakurskiy, V. I. Filippov, V. I. Ruzhickiy, N. V. Klenov, I. I. Soloviev, M. Y. Kupriyanov, and A. A. Golubov, Current-phase relations in SIsFS junctions in the vicinity of $0-\pi$ transition, Phys. Rev. B 95, 094522 (2017).

[33] L. Trifunovic, Long-Range Superharmonic Josephson Current, Phys. Rev. Lett. 107, 047001 (2011).

[34] C. Richard, M. Houzet, and J. S. Meyer, Superharmonic Long-Range Triplet Current in a Diffusive Josephson Junction, Phys. Rev. Lett. 110, 217004 (2013).

[35] A. Pal, Z. H. Barber, J. W. A. Robinson, and M. G. Blamire, Pure second harmonic current-phase relation in spin filter Josephson junctions, Nat. Commun. 5, 3340 (2014).

[36] B. Baek, M. L. Schneider, M. R. Pufall, and W. H. Rippard, Anomalous supercurrent modulation in Josephson junctions with Ni-based barriers, IEEE Trans. Appl. Supercond. 28, 1800705 (2018).

[37] M. J. A. Stoutimore, A. N. Rossolenko, V. V. Bolginov, V. A. Oboznov, A. Y. Rusanov, N. Pugach, S. M. Frolov, V. V. Ryazanov, and D. J. V. Harlingen, SecondHarmonic Current-Phase Relation in Josephson Junctions with Ferromagnetic Barriers, Phys. Rev. Lett. 121, 177702 (2018).

[38] N. G. Pugach, E. Goldobin, R. Kleiner, and D. Koelle, Method for reliable realization of a $\varphi$ josephson junction, Phys. Rev. B 81, 104513 (2010).

[39] N. V. Klenov, N. G. Pugach, A. V. Sharafiev, S. V. Bakurskiy, and V. K. Kornev, Josephson junctions with nonsinusoidal current-phase relations based on heterostructures with a ferromagnetic spacer and their applications, Phys. Solid State 52, 2246 (2010).

[40] H. Sickinger, A. Lipman, M. Weides, R. G. Mints, H. Kohlstedt, D. Koelle, R. Kleiner, and E. Goldobin, Experimental Evidence of a $\varphi$ Josephson Junction, Phys. Rev. Lett. 109, 107002 (2012).

[41] E. Goldobin, H. Sickinger, M. Weides, N. Ruppelt, H. Kohlstedt, R. Kleiner, and D. Koelle, Memory cell based on a $\varphi$ Josephson junction, Appl. Phys. Lett. 102, 242602 (2013).

[42] S. V. Bakurskiy, N. V. Klenov, T. Y. Karminskaya, M. Y. Kupriyanov, and A. A. Golubov, Josephson $\varphi$-junctions based on structures with complex normal/ferromagnet bilayer, Supercond. Sci. Technol. 26, 015005 (2013).

[43] E. Goldobin, D. Koelle, and R. Kleiner, Tunable $\pm \varphi, \varphi_{0}$, and $\varphi_{0} \pm \varphi$ Josephson junction, Phys. Rev. B 91, 214511 (2015).

[44] W. C. Stewart, Current--voltage characteristics of Josephson junctions, Appl. Phys. Lett. 12, 277 (1968).

[45] A. F. Kirichenko, Universal delay-insensitive logic cell, US patent 6,486,694 B1 (2002).

[46] D. E. Kirichenko, S. Sarwana, and A. F. Kirichenko, Zero static power dissipation biasing of RSFQ circuits, IEEE Trans. Appl. Supercond. 21, 776 (2011).

[47] A. F. Kirichenko, I. V. Vernik, J. A. Vivalda, R. T. Hunt, and D. T. Yohannes, ERSFQ 8-bit parallel adders as a process benchmark, IEEE Trans. Appl. Supercond. 25, 1300505 (2015).

[48] A. Shukla, B. Chonigman, A. Sahu, D. Kirichenko, A. Inamdar, and D. Gupta, in IEEE Transactions on Applied Superconductivity (2019), Vol. 29, p. 1.

[49] T. Jabbari, G. Krylov, S. Whiteley, E. Mlinar, J. Kawa, and E. G. Friedman, in IEEE Transactions on Applied Superconductivity (2019), Vol. 29, p. 1. 